\title{
Radiation Spectrum of the Hydrogen Atom with the Account of Motion of Its Electron in the Extra Space
}

\author{
Igor Alekseevich Urusovskii \\ N. N. Andreyev Acoustics Institute, Moscow, Russia \\ Email: urusovskii_ia@mail.ru
}

How to cite this paper: Urusovskii, I.A. (2017) Radiation Spectrum of the Hydrogen Atom with the Account of Motion of Its Electron in the Extra Space. Journal of Modern Physics, 8, 1961-1978. https://doi.org/10.4236/jmp.2017.812118

Received: October 17, 2017

Accepted: November 21, 2017

Published: November 24, 017

Copyright $\odot 2017$ by author and Scientific Research Publishing Inc. This work is licensed under the Creative Commons Attribution International License (CC BY 4.0).

http://creativecommons.org/licenses/by/4.0/

\section{(c) (i) Open Access}

\begin{abstract}
A multidimensional interpretation of the emission spectrum of a hydrogen atom for the circular orbits of its electron is given. It is shown that the discreteness of the radiation frequencies and the angular momentum of an electron for quasi-Bohr orbits are due to the periodicity of the motion, both in the projection to the observed three-dimensional space, permitting motion by inertia, and on additional space. The fine structure constant is represented as a simple function of the ratio of the radii of the orbits in the complementary and observed subspaces of the total space. The balance of forces acting on an electron in the corresponding subspaces allows one to find the electron $\mathrm{Ha}$ miltonian in orbits, the work of exit of the electron from an atom, and the emission spectrum of the atom.
\end{abstract}

\section{Keywords}

Radiation Spectrum, Fine Structure Constant

\section{Introduction}

Observations show that our three-dimensional Universe is isotropic and homogeneous at distances of more than 300 million light-years [1]. This means that on such a scale, the three-dimensional Universe has no distinguished points, its curvature at all points is the same, and therefore it is a three-dimensional sphere and can be and expand only in the space of a higher number of spatial dimensions.

The scale of inhomogeneities in the Universe is 100,000 times smaller than the characteristic size of the Metagalaxy (the observable part of the Universe). $6 \mathrm{D}$-cosmology gives the value of 3980 Megaparsec for the radius of the Metaga- 
laxy [2]. The length of a large circle of the Metagalaxy passing through the points of its boundary (the particle horizon) can be taken as such a characteristic size. Then the size of the inhomogeneities in the Metagalaxy is $2 \pi 3980 / 100,000=$ $0.25 \mathrm{Mpc}$, which is 815,646 light years, which is approximately equal to the average distance between galaxies. The principal curvature of the Universe as a three-dimensional sphere in any plane section passing through the center of our $3 \mathrm{D}$-sphere is equal to unity, divided by the radius of this sphere. Today's radius of the Universe is $7100 \mathrm{Mpc}$ [2]. With the expansion of the Universe, its curvature comparatively rapidly tends to zero, and the space of the Universe becomes locally Euclidean, which differs markedly from Euclidean space only at cosmological distances. Therefore, in physical theories that are not related to cosmology explicitly, the space can be considered as Euclidean one, but it is necessary to take into account the motion of elementary particles in the additional space.

Geometric and physical characteristics of the three-dimensional universe are found by the formulas of the cosmological model under consideration, based on the principle of simplicity, for fixed free parameters of the theory. These parameters are chosen so that the deviations of all compared quantities from each other are minimal. The simplest object in the six-dimensional Euclidean space is the five-dimensional sphere of perturbations in this space. The intersections of the three expanding five-dimensional spheres are three expanding four-dimensional spheres, the mutual intersections of which form three expanding three-dimensional spheres. One of them is our three-dimensional Universe [2].

If formulas of Newtonian mechanics are attributed not to the three-dimensional but to the six-dimensional space, then formulas of the special theory of relativity and quantum mechanics are obtained, provided that the proper time of any elementary particle is proportional to the path traversed by it in the additional space, and the velocity of all particles in the total space is the same in magnitude and is equal to the upper limit of the speed of light $c$ [3].

In order the observed interaction of elementary particles to occur, the particles should be kept in sufficient proximity to the three-dimensional Universe in the Compton neighborhood of the closest points of our Universe by cosmological forces perpendicular to the Universe. These are forces of the Lorentz force type, in which the particle's mass plays the role of the charge, in a magnetic field oriented along the radius of the Universe [3] [4] [5]. These forces give to the space waveguide properties, including dispersion [5]. Without such forces, the formation of macroscopic bodies would be impossible.

A simple interpretation of the spin and isotopic spin requires at least three additional spatial dimensions. This gives a simple interpretation of the Heisenberg uncertainty relation, de Broglie waves, the Klein-Gordon equation, the intrinsic magnetic moment of the electron, CPT symmetry, and the dispersion of the photon velocity in vacuum [3] [4] [5]. In the six-dimensional space, spin and isospin are treated as projections of the total angular momentum, respectively, 
onto the observed and additional subspace, and the proper magnetic moment as the result of rotation of the charge in the complementary subspace in the orbit of the Compton radius.

The potential energy of a particle is the energy of its motion in an additional subspace, and the nuclear reaction is a redistribution of the motion of particles between two subspaces.

\section{The Motion of Elementary Particles in a Complete Space with a Fundamental Velocity Equal to the Upper Limit of the Speed of Light}

The dispersion equation for an acoustic waveguide, for an electromagnetic waveguide and for de Broglie waves is one and the same: $v_{\phi} v_{g}=c^{2}$ where the $v_{\phi}$ is phase velocity of waves, $v_{g}$ the group velocity (for de Broglie waves is equal to the velocity of the corresponding particle), $c$ the velocity of waves in a free medium (the speed of sound in the first case and light in the other two). The main characteristic of any waveguide is that it has finite transverse dimensions. They also determine the dispersion of waves. This indicates that the part of the space we are dealing with in experiments and observations is three-dimensional only approximately and has small (Compton) dimensions in an additional subspace to which all directions in the observed three-dimensional subspace are perpendicular.

In the waveguide, the rays experience successive reflections from its boundaries, or they move along helical lines oriented along the axis of the waveguide. Therefore, along the waveguide, the photons and other elementary particles move with a lower velocity $v_{g}=c \sin \theta$ than the phase fronts of the normal waves moving along the rays with velocity $c$. Here $\theta$ is the angle between the ray and the cross section of the waveguide. The points of intersection of the front with the generatrix of the waveguide move with a phase velocity $v_{\phi}=c / \sin \theta$ exceeding the velocity $c$, whence the dispersion law $v_{\phi} v_{g}=c^{2}$ follows.

The proposed interpretation of the motion of elementary particles is based on the principle of simplicity [6], giving preference to that of competing hypotheses, which is based on a smaller number of postulates, i.e. more simple. It goes back to the assertion of Einstein that "the nature saves on principles" and to the idea of F. Klein [7] [8] about the motion of particles with the speed of light in a multidimensional space, also fitting into the principle of simplicity and its concretizing.

The total space is assumed to be six-dimensional one, since a simple interpretation of the spin and isospin of an electron and other particles is possible. Spin is the projection of the total angular momentum of an elementary particle into an observable three-dimensional subspace $X$ in which the motion by inertia is possible. In quantum mechanics, spin is treated as the result of an imaginary rotation of a particle. Isospin is the projection of the total momentum of a particle 
onto the additional subspace. Despite the impossibility of treating spin and isospin in the framework of classical physics, they manifest themselves in the interactions of particles and can be measured.

The first substantiation of the six-dimensionality of space was given in [9], where 3 time coordinates relate to the three-dimensional time and 3 spatial ones to the observed three-dimensional subspace. In [9] theoretical values of fundamental physical constants were obtained, for the seven most common of which, obviously not being a combination of each other, the first 36 significant figures are confirmed by experiment, so the probability of random coincidence with experimental data does not exceed $10^{-36}(!)$.

The position of the particles in $R_{6}$ is described by six coordinates. The observer, however, fixes the particles in the projection onto the subspace X. Even a particle at rest in the inertial system of the observer moves in a complete space with the fundamental velocity $c$ equal to the upper limit of the speed of light. In the simplest case, this motion occurs along a circle located in one of the planes of an additional three-dimensional subspace $y_{1}, y_{2}, y_{3}(Y)$, with the center located in X for $y_{1}=y_{2}=y_{3}=0$. In any other inertial frame of reference, the particle in question moves in $R_{6}$ along a geodesic helical line, located on a cylindrical surface, enveloping the geodesics, which for brevity we will call the tube of motion.

\section{Lorentz Transformations and Formulas of Quantum Mechanics as the Result of Taking into Account the Motion of Elementary Particles in the Additional Space}

The natural measure of the particle's proper time is the path length of the particle in the additional subspace $Y$ around the tube axis. In general, this length is proportional to $|\cos \theta|$, where $\theta$ is the angle of inclination of the helical line to the directrix of the tube of motion (Figure 1), $a=\hbar /(m c)$ is the radius of the tube. Therefore, if the particle makes one revolution for its proper time $\tau$, then by the clock of the observer at rest, relative to which the particle moves along the tube at a speed $v=c \sin \theta$, this will happen in time $t=\tau /|\cos \theta|$. Here

$$
\sin \theta=v / c, \cos \theta= \pm \sqrt{1-(v / c)^{2}},
$$

where the upper sign in front of the square root refers to a particle rotating around the axis of the tube in the positive direction, the lower one to its antiparticle moving in the opposite direction. This choice of sign corresponds to the following relation between the intervals of the proper time $d \tau$ of the particle (or antiparticle) and the time $d t$ of the observer at rest:

$$
d t= \pm d \tau / \cos \theta=d \tau / \sqrt{1-(v / c)^{2}} .
$$

A particle moving with a velocity $c$ in a fixed reference frame $(K)$ at an angle $\theta$ to the directrix of the tube has a velocity component along the directrix, equal to $c \cos \theta$. The course of the particle's proper time from the point of 


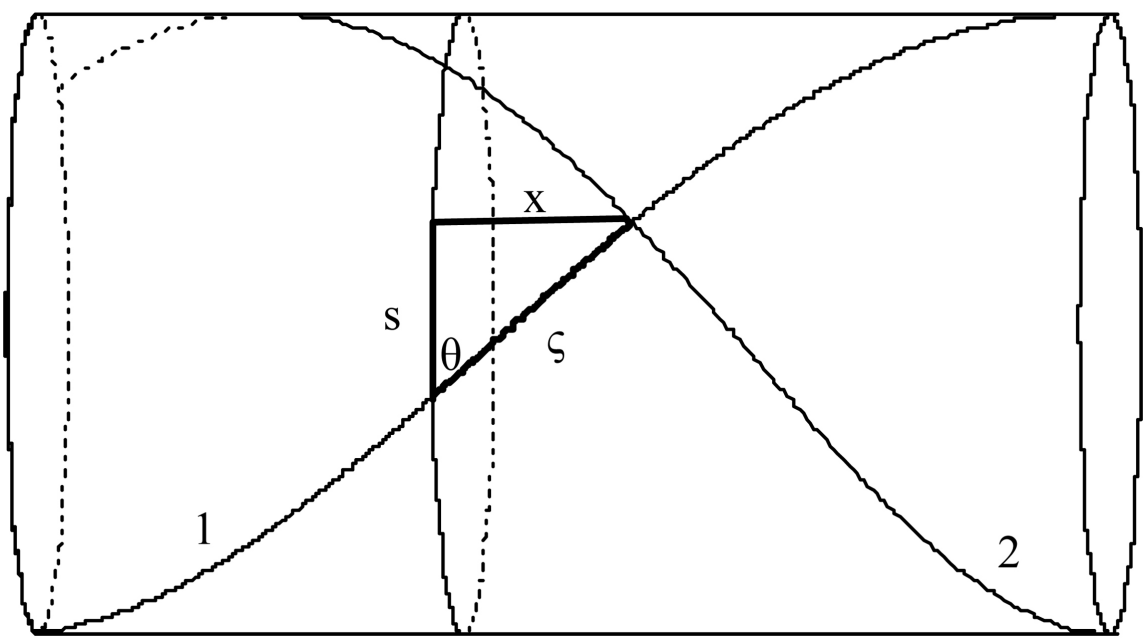

Figure 1. Helical trajectory of an elementary particle in a mixed subspace $x y_{2} y_{3}: 1$ is the helical trajectory of a particle moving with a fundamental velocity $c$ in a six-dimensional space along the surface of a cylinder of Compton radius $a=\hbar /(m c)$ with the angle $\theta$ of inclination of this helical line to the directrix of the tube passing in the subspace $Y ; 2$ is other helical line passing through the particle at a right angle to its trajectory, of the same proper time of the particle, moving along the cylinder with the velocity of the de Broglie waves; its step is equal to the wavelength of de Broglie.

view of the observer at rest is slowed according to (2) also in proportion to $\cos \theta$. Therefore, the particle in question also moves along the directrix with velocity $C$ in its proper frame of reference $\left(K^{\prime}\right)$.

A particle at rest in the system $K$ moves along the directrix at the speed $c$. In this case, it displaces along the directrix for its proper time $d \tau$ to an interval ds equal to

$$
d s= \pm c d \tau
$$

The momentum (the amount of motion) of this particle is a vector directed along the tangent to the directrix at the point at which the particle is at this moment, and equal in magnitude to $m c$, the product of the particle's mass $m$ by its velocity in total space. This value is equal to the momentum at rest of relativistic mechanics. The energy at rest $E_{0}$ is by definition equal to the product of the momentum at rest by the velocity of the particle along the directrix, so that $E_{0}=m c^{2}$. This Einstein formula relates the mass and latent energy of the motion of an elementary particle in the additional subspace.

In the general case, the total momentum of a particle is a vector directed along the tangent to its helical trajectory on the tube of motion and equal in magnitude to the product of the mass of the particle by the ratio of the path it traveled

$$
d \varsigma=c d t
$$

in full space to the time spent on this path:

$$
p=m \frac{d \varsigma}{d \tau}=\frac{m c}{|\cos \theta|}=\frac{m c}{\sqrt{1-(v / c)^{2}}} .
$$


This is a well-known relativistic formula for the total particle momentum [10]. The projections $p_{X}$ and $p_{y}$ of the total momentum on the generatrix and the tube directrix are equal, respectively,

$$
p_{X}= \pm m c \tan \theta=m v / \sqrt{1-(v / c)^{2}}, p_{y}= \pm m c
$$

These projections are called in relativistic mechanics, respectively, the coordinate and time components of the particle's 4-momentum. The total energy $E$ of the particle motion is equal to the product of the total momentum $p$ by the speed $c$ of motion along the helical line:

$$
E=p c=\frac{m c^{2}}{|\cos \theta|}=\frac{m c^{2}}{\sqrt{1-(v / c)^{2}}} .
$$

We assume that the oppositely charged particles rotate about the axis of the tube of motion in opposite directions. Particles and antiparticles have opposite charges and rotate in opposite directions. When the time reverses, the particle must move backwards along its trajectory (the helical line) and therefore rotate in the opposite direction. Hence, the sign of its charge will have to change to the opposite, so that the particle will have to turn into its antiparticle. In this case, the motion of the particle will be mirrored. The totality of all these properties of particles and antiparticles constitute the content of the CPT theorem (here C means a change in the sign of the charge, $\mathrm{P}$ is the reflection in the mirror, and $\mathrm{T}$ is the inversion of time).

The displacement of the particle to the interval $d s$ along the directrix of the tube of motion and the corresponding rotation to the central angle $d \phi=d s / a$, where $a$ is the radius of the tube cross section, are invariants: the angle of rotation $\phi$ of the particle around the axis of the tube does not depend on the speed of the observer relative to the given particle.

Let us designate $d x$ in the frame of reference $K$ the projection of the displacement $d \varsigma$ of the particle along the surface of the tube onto its generatrix. By the Pythagorean theorem for a right-angled triangle on the surface of the tube shown in Figure 2, we obtain an expression for the interval $(d s)^{2}=(c d t)^{2}-(d x)^{2}$, which is fundamental one in the theory of relativity.

Projecting the sides shown in Figure 1 of a right-angled triangle onto the trajectory of the particle, we find

$$
s \cos \theta+x \sin \theta=\varsigma .
$$

We choose the initial conditions as $x=s=0$ for $t=\tau=0$. In accordance with (3) and (4), they correspond to

$$
s= \pm c \tau, \varsigma=c t .
$$

Substituting (1) and (9) in (8), we obtain the Lorentz transformation in time

$$
\tau= \pm[t-(x / c) \sin \theta] / \cos \theta=\left[t-\left(x v / c^{2}\right)\right] / \sqrt{1-(v / c)^{2}} .
$$

A similar reasoning applied to the frame of reference $K^{\prime}$, taking into account the fact that the system $K$ moves relative to the particle under consideration at 


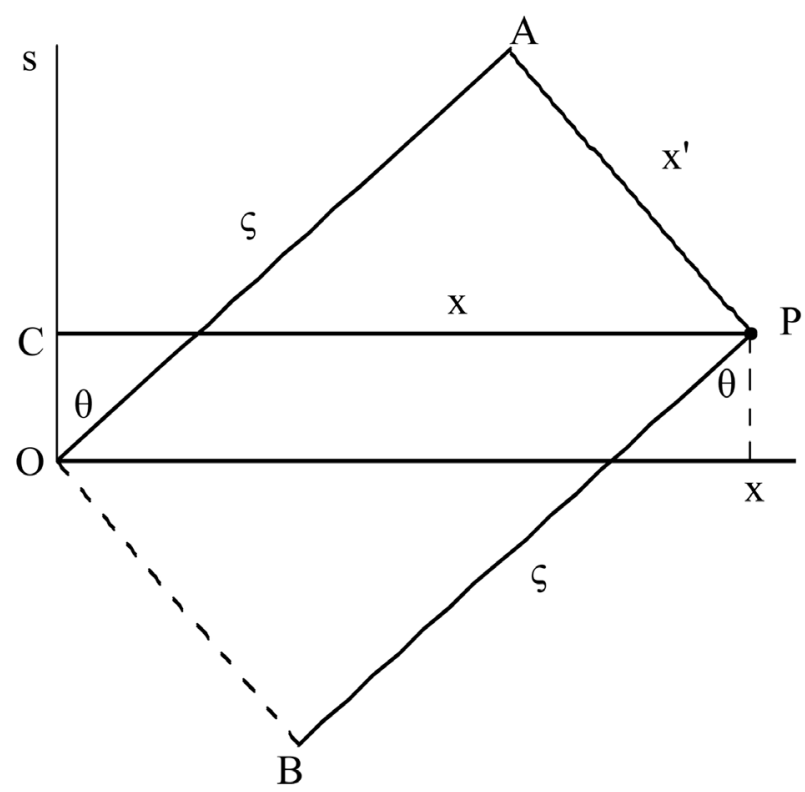

Figure 2. The developed view of the tube of motion and the particle trajectory: $x$ and $s$ (interval) is the coordinates of the particle along the generatrix and directrix of the cylinder in the fixed reference frame; $x^{\prime}$ is the same along the generatrix in the frame of reference associated with the particle. $B P=O A=\varsigma, C P=x, A P=O B=x^{\prime}, \quad s=\varsigma \cos \theta$.

a speed $-v$, leads to an inverse transformation

$$
t= \pm\left[\tau+\left(x^{\prime} / c\right) \sin \theta\right] / \cos \theta=\left[\tau+\left(x^{\prime} v / c^{2}\right)\right] / \sqrt{1-(v / c)^{2}}
$$

where $x^{\prime}$ is the coordinate along the generatrix in the system $K^{\prime}$. The transition from the system $K$ to the system $K^{\prime}$ corresponds to a turn through an angle $-\theta$ around the point $x=s=0$ of the coordinate grid $x, s$ on the surface of the motion tube, together with the trajectories of the particles on it, which translates the helical trajectory into directrix of the tube.

The trajectories that make up the angle $\theta$ with the directrix in the reference frame $K$ of the fixed (in $X$ ) observer are lines of constant values of the coordinate $x^{\prime}$ in the system $K^{\prime}$. The coordinate $x^{\prime}$ of the particle $P$ moving along the tube with the corresponding velocity $v$ is measured along a helical line perpendicular to the trajectory of the particle. In Figure 2, depicting the motion tube of a particle in developed view, $x^{\prime}(t)$ is represented by a segment $A P$, and $x(t)$ by a segment $C P$. Projecting the segments $A O$ and $A P$ onto the segment $C P$, we obtain for $\cos \theta>0: x^{\prime} \cos \theta+\varsigma \sin \theta=x$ or, taking into account (1), $x^{\prime}=(x-v t) / \sqrt{1-(v / c)^{2}}$.

The proper length of a moving rigid scale is the difference in the coordinates $x^{\prime}$ of its ends, and in the system $K$ is equal to the length of a segment of the helical line orthogonal to the trajectories of particles moving with it, a line of the same time of the reference system $K^{\prime}$ associated with the particles, between the normal sections of the motion tube corresponding to these ends. The length of 
the same moving scale in a fixed reference system is the difference in the coordinates $x$ of its ends, equal to the distance along the generatrix between the indicated sections, which is $1 / \cos \theta$ times less than the proper length. Thus, the Lorentz contraction of moving scales is the result of the projection of lengths in a multidimensional space onto a three-dimensional space. The spuriousness of spatially separated events in one frame of reference when they are simultaneously in another is interpreted by the non-parallelism of the helical lines of the same proper time in moving frames relative to one another.

The above interpretation of Formula (2) remains valid even with the curvilinear axis of the motion tube, since even then all normal sections of the motion tube are perpendicular to all directions in the subspace $X$ containing the axis of the tube.

By virtue of the principle of identical properties of the basic properties of matter and light, the rest energy $m c^{2}$ must also equal $h v_{0}$ where $v_{0}$ is the frequency of rotation of the particle around the axis of the motion tube with velocity $c$ in the additional subspace $Y, h$ is Planck's constant, so that $m c^{2}=h v_{0}$. Hence it follows that the radius of the tube is equal $a=\hbar / m c$, and the length of the directrix is equal to the Compton wavelength $2 \pi a=h / m c$, which corresponds to the period $h$ of the coordinate of action in 5-optics [11].

Located on the same tube of motion, a helical line passing through the particle and perpendicular to the helical trajectory of the particle is the line of the same current time of the reference frame $K^{\prime}$. This line moves along the tube with the velocity $V_{\phi}=c / \sin \theta=c^{2} / v$ of the de Broglie waves, where $v$ is the particle velocity in the subspace $X$. The interference of these waves describes, in particular, the scattering of electrons that strike a crystal with velocity $v$. The pitch $\ell$ of this helical line is equal to the de Broglie wave $\ell=2 \pi a(|\cot \theta|)=\frac{h}{m c}|\cot \theta|=\frac{h}{p_{x}}=\frac{h}{|m v|} \sqrt{1-(v / c)^{2}}$, as can be seen from (6) and Figure 1. The angular coordinate $s / a$ of the helical line described by formulas (8) and (9) is $\frac{s}{a}=\frac{\varsigma}{a \cos \theta}-\frac{x}{a} \tan \theta=t \frac{m c^{2}}{\hbar \cos \theta}-x \frac{m c}{\hbar} \tan \theta=\frac{c t-x \sin \theta}{a \cos \theta}$, where $\hbar=h / 2 \pi$. From this, (6) and (7) it is clear that this angular coordinate is equal to the phase $\pm\left[E t-p_{x}(x / \hbar)\right]$ of the de Broglie wave. At the location $x=v t$ of the particle, this phase is the angle of turn of the particle itself on the tube of motion. We note that the function $\Psi=\exp ($ is $/ a)$ satisfies the Klein-Gordon equation $a^{2} \frac{\partial^{2} \Psi}{\partial x^{2}}-a^{2} \frac{\partial^{2} \Psi}{\partial \varsigma^{2}}=\Psi$ of relativistic quantum mechanics [4]. This can be seen from the relations $\Psi_{\varsigma}=\Psi \frac{i}{a} s_{\varsigma}, \quad-a^{2} \Psi_{\varsigma \varsigma}=\Psi s_{\varsigma}^{2}, \quad s=\frac{\varsigma}{\cos \theta}-x \tan \theta$, $s_{\varsigma}^{2}=\frac{1}{\cos ^{2} \theta}, s_{x}^{2}=\tan ^{2} \theta, a^{2} \Psi_{x x}=-\Psi s_{x}^{2}, a^{2} \Psi_{x x}=-\Psi s_{x}^{2}, s_{\varsigma}^{2}-s_{x}^{2}=1$.

The angular momentum $\boldsymbol{J}$ of the particle is equal to the vector product of the momentum and the radius vector of the particle. The component of the radius vector along the axis of the particle motion tube is parallel to the momen- 
tum and therefore does not gives a contribution in $\boldsymbol{J}$. Therefore, for a particle moving in a six-dimensional space along a helical line, and therefore rectilinearly in the projection onto a subspace $X, \boldsymbol{J}$ is the vector product of the projections of the momentum and the radius vector of the particle onto the subspace $Y$. The magnitude of the angular momentum is $J=|\boldsymbol{J}|=\left|p_{y} a\right|=m c \hbar / m c=\hbar$.

This formula leaves a known arbitrariness in the orientation of the vector $\boldsymbol{J}$ in six-dimensional space: it can be oriented along any directions of the four-dimensional subspace, all directions in which are perpendicular to the plane of rotation of the particle in $Y$, i.e., to the cross section of the motion tube. The angular momentum $\boldsymbol{J}$ in the general case has four nonzero components in directions perpendicular to each other and the plane of rotation of the particle. In the case of rotation in a plane $y_{2}, y_{3}$, such components will be the components $J_{1}, J_{2}, J_{3}, J_{4}$ along the axes $x_{1}, x_{2}, x_{3}, y_{1}$, respectively. Wherein, $J=\left(J_{1}^{2}+J_{2}^{2}+J_{3}^{2}+J_{4}^{2}\right)^{1 / 2}=\hbar$. The components $J_{1}, J_{2}, J_{3}$ are projections of the vector $\boldsymbol{J}$ onto the subspace $X$ and form a spin, and the component $J_{4}$ is the projection of the vector $\boldsymbol{J}$ onto the subspace $Y$ and forms an isospin.

According to (6), $p_{y}$ does not depend on the velocity $v$, therefore, spin and isospin do not depend on $v$ and are not affected by Lorentz transformations.

The vector $\boldsymbol{J}$, which remains perpendicular to the plane of rotation of the particle, has three degrees of freedom and can be arbitrarily oriented along four coordinate axes. The simplest methods of its orientation are:

1) The vector $\boldsymbol{J}$ does not have components in $X$ and is entirely located in $Y$; then the spin of the particle is zero, and the isospin is equal to unity, as in the case of the $\pi$-meson.

2) The vector $\boldsymbol{J}$ does not have components in $Y$ and is entirely located in $X$; then the spin of the particle is equal to unity, and the isospin to zero, to this case is the photon;

3) Particles with spin $1 / 2$ correspond to a uniform distribution of the components of the vector $\boldsymbol{J}$ along four axes perpendicular to each other and to the plane of rotation of the particle. Then these components are equal to $+\hbar / 2$ or $-\hbar / 2$, and the sum of the squares of these components in $X$ of is equal to $(3 / 4) \hbar^{2}$, which coincides with the square of the "total" (in three-dimensional space) quantum momentum of the particle. The orientations of the vector $\boldsymbol{J}$, obtained from the previous by admissible rotations, leaving one or two given components unchanged also belong to this case. So, if one of the components of the vector $\boldsymbol{J}$ in $X$ and one of its components in $Y$ have a fixed value $+\hbar / 2$ or $-\hbar / 2$, then the vector $\boldsymbol{J}$ still has the possibility of rotation around two corresponding axes. In this case, the two components of the vector $\boldsymbol{J}$ that remain uncommitted do not have a certain value (a situation typical for quantum mechanics, where a fixed value of the quantity is the exception rather than the rule). For an equiprobably permissible orientations of this vector, the rms values of these components are equal to $\hbar / 2$. The change in the direction of rotation 
about the axis of the tube of movement to the opposite also changes the signs of the components to opposite ones and corresponds to the transition to the antiparticle.

The Heisenberg uncertainty relations are related to the uncertainty of the coordinates and momenta of elementary particles in $Y$, which obey these relations. Indeed, let the directrix of the tube be in the plane $y_{2}, y_{3}$. Then the coordinates of the particle along the axes $y_{2}, y_{3}$ and the projections of the particle momentum on these axes are, respectively, $y_{2}=\frac{\hbar}{m c} \cos \phi$, $y_{3}=\frac{\hbar}{m c} \sin \phi, \quad p_{y 2}=-m c \sin \phi, \quad p_{y 3}=m c \cos \phi$, where $\phi$ is the angle of turn of the particle around the axis of the tube measured from the axis $y_{2}$.

The average on $\phi$ values of the coordinates and projections of the pulses are zero, and their mean squares are respectively equal to $\left\langle y_{2}^{2}\right\rangle=\left\langle y_{3}^{2}\right\rangle=\frac{1}{2}\left(\frac{\hbar}{m c}\right)^{2}$, $\left\langle p_{y 2}^{2}\right\rangle=\left\langle p_{y 3}^{2}\right\rangle=\frac{1}{2}(m c)^{2}$, from which the desired relations follow $\left\langle p_{y 2}^{2}\right\rangle \cdot\left\langle y_{2}^{2}\right\rangle=\left\langle p_{y 3}^{2}\right\rangle \cdot\left\langle y_{3}^{2}\right\rangle=\frac{1}{4} \hbar^{2} \quad[4]$.

The question is interesting: why does the magnitude of the angular momentum and its components in $X$ and $Y$, that is, the spin and isospin, not depend on the mass of the elementary particle? In the six-dimensional treatment, the answer is obvious: the momentum is proportional to the mass of the particle, and the radius of the Compton orbit of such a particle is inversely proportional to this mass, and therefore the product of the momentum and the radius of the orbit does not depend on the mass.

The proper magnetic moment $\boldsymbol{\mu}$ of a charged elementary particle is determined similarly to the proper moment of the pulse $\boldsymbol{J}$. Namely, according to the well-known electrodynamics formula [10] [12]

$$
\boldsymbol{\mu}=\frac{e}{2 c}[\boldsymbol{R c}],
$$

where $\boldsymbol{R}$ is the radius vector of the particle, $\boldsymbol{c}$ is its velocity vector in the complete space, $[\boldsymbol{R} \boldsymbol{c}]$ is the vector product of the vectors $\boldsymbol{R}$ and $\boldsymbol{c}, e$ the particle charge. Since the contribution to the vector product in (10) is given only by the projection $\boldsymbol{a}$ of the radius vector $\boldsymbol{R}$ on $Y$, the vector $\boldsymbol{\mu}$ equals $\frac{e}{2 c}[\boldsymbol{a c}]$. Hence, taking into account the mutual perpendicularity of the vectors $\boldsymbol{a}$ and $\boldsymbol{c}$, and denoting $|\boldsymbol{a}|=a$ and $|\boldsymbol{c}|=c$, we obtain the value of the proper magnetic moment $\mu$ of the particle, which, as it turns out, is equal to the Bohr magneton:

$$
\mu=\frac{|e| a}{2}=\frac{|e| \hbar}{2 m c}=\mu_{B}
$$

In the simplest case, when the vector $\boldsymbol{\mu}$ does not have components in the 
subspace $Y$, its components in $X$ define a three-dimensional vector whose magnitude is equal to the Bohr magneton. The projection of the magnetic moment on an arbitrarily chosen direction (the quantization axis) in the subspace $X$ can have a fixed value $\left(\mu_{x}\right)$ only in the case when the projection of the proper angular momentum $J_{x}$ on this direction also has a fixed value. According to (11), in this case, $\mu_{x}= \pm \mu_{B}$. For a uniform distribution of the components of the proper angular momentum along four axes perpendicular to one another and the plane of rotation of the particle in $Y$, in the case under consideration $J_{x}= \pm m c a / 2$, which is equal to $+1 / 2$ or $-1 / 2$ (in units $\hbar$ ) in accordance with the data of Stern and Gerlach experiment.

\section{The Condition for the Periodicity of the Motion of an Electron in a Complete Space in a Hydrogen Atom and the Balance of Forces Acting on an Electron}

In addition to rotation in $Y$ in orbits of the Compton radius, the electrons in the atom move in $X$ in orbits much larger (by a factor of hundreds) radius. The dimensions of the atoms are equal to the dimensions of their external electron orbits in $X$. Let us show that the condition for the periodicity of the motion of an electron in a hydrogen atom determines a quasi-Bohr orbits [13].

In the hydrogen atom, the distance of the electron in the complete space from the center of the atom has the form $R=\sqrt{r^{2}+a^{2}}$, where $r$ and $a$ are the radii of the circles along which the electron rotates, respectively, in the projection onto the subspaces $X$ and $Y$. The radius $a$ with increasing $r$ asymptotically tends to $a_{\infty}=\hbar /(m c)$.

The tube of stationary motion of an electron in a hydrogen atom in the simplest case is a closed surface, which in the projection to $X$ is a circle with the center in the nucleus of the atom. This surface, in contrast to the usual torus, preserves the properties of the cylinder, since all its points are separated from the center of rotation by the same distance in the total space. The condition of periodicity of motion implies the closure of the helical line of the same current proper time of the electron on the tube of its motion. Suppose that when the angular coordinate in $X$ of the current point of this helical line makes one complete revolution (changes by $2 \pi$ ), its angular coordinate in $Y$, corresponding to $n$-th orbit makes $n$ revolutions. In this case, $\sin \theta=v / c$ has a form $\sin \theta_{n}=v_{n} / c$,

$$
\tan \theta_{n}=\frac{v_{n} / c}{\sqrt{1-\left(v_{n} / c\right)^{2}}}=n \frac{a_{n}}{r_{n}} .
$$

Whence

$$
\left(v_{n} / c\right)^{2}=\left(\frac{n a_{n}}{r_{n}}\right)^{2} \frac{1}{1+\left(n a_{n} / r_{n}\right)^{2}},
$$

where $r_{n}$ is the radius of the orbit in $X, n=1,2,3, \cdots$ the orbit number in ascending order $r_{n}, \theta_{n}$ the angle of inclination of the trajectory of the electron 
to the normal section of the tube, $a_{n}$ the radius of the tube in $Y, a_{\infty}=\hbar /(m c)$, $m$ the mass of the electron. Since $a_{n} \approx \hbar /(m c)$, from Formula (13) it follows $m v_{n} r_{n} \sqrt{1+\left(\frac{n \hbar}{m c r_{n}}\right)^{2}} \approx n \hbar$. In the non-relativistic case, the last formula reduces to the classical expression from Bohr's theory for the angular momentum $m v_{n} r_{n} \approx n \hbar$.

$$
\begin{aligned}
& \text { Substitution of Formula (12) into identity } \frac{1}{\sqrt{1+\tan ^{2} \theta_{n}}}=\cos \theta_{n} \text { gives } \\
& \qquad \frac{1}{\cos \theta_{n}}=\sqrt{1+\left(n a_{n} / r_{n}\right)^{2}} \\
& \sin ^{2} \theta_{n}=\frac{\left(n a_{n} / r_{n}\right)^{2}}{1+\left(n a_{n} / r_{n}\right)^{2}}, \frac{\sin ^{2} \theta_{n}}{\cos \theta_{n}}=\frac{\left(n a_{n} / r_{n}\right)^{2}}{\sqrt{1+\left(n a_{n} / r_{n}\right)^{2}}} .
\end{aligned}
$$

The Hamiltonian of an electron in the $n$-th orbit with (14) taken into account has the form: $H_{n}=m c^{2} \sqrt{1+\left(n a_{n} / r_{n}\right)^{2}}+V_{n}$. It is equal to the sum of the energy of motion in total space and potential energy $V_{n}$ and represents a multidimensional structure. The Coulomb potential has so far remained three-dimensional one also in standard quantum mechanics, where in deriving the fine structure formula [13], the distance between the electron and the nucleus of the atom was assumed to be equal $r_{n}$ instead of the correct one $R_{n}=\sqrt{r_{n}^{2}+a_{n}^{2}}$. In this case $V_{n}=-e^{2} / r_{n}$, and the electric force has the form $\frac{e^{2}}{r_{n}^{2}} \sqrt{1-\frac{v_{n}^{2}}{c^{2}}}=\frac{e^{2}}{r_{n}^{2}} \cos \theta_{n}$. It is opposed by an equal in magnitude centrifugal force

$$
p_{x} \frac{v}{r}=m v_{n}^{2} /\left(r_{n} \sqrt{1-\frac{v_{n}^{2}}{c^{2}}}\right)=m c^{2} \sin ^{2} \theta_{n} /\left(r_{n} \cos \theta_{n}\right)=m c^{2} \frac{1}{r_{n}}\left(\frac{n a_{n}}{r_{n}}\right)^{2} \cos \theta_{n} .
$$

In this case, the balance of forces has the form $e^{2} / r_{n}=m c^{2} \tan ^{2} \theta_{n}$ or, taking into account (12) and $e^{2}=\alpha a_{\infty} m c^{2}$, where $\alpha=e^{2} /(\hbar c)=1 / 137.035999710$ is the fine structure constant, the form $e^{2} \frac{1}{r_{n}}=m c^{2}\left(\frac{n a_{n}}{r_{n}}\right)^{2}$,

$$
\begin{aligned}
\alpha a_{\infty} \frac{1}{r_{n}}=\tan ^{2} \theta_{n}=\frac{n^{2} a_{n}^{2}}{r_{n}^{2}} \text { or } \frac{1}{r_{n}}= & \frac{\alpha a_{\infty}}{n^{2} a_{n}^{2}}, \\
\frac{n a_{n}}{r_{n}} & =\frac{\alpha a_{\infty}}{n a_{n}} .
\end{aligned}
$$

From this and from (14) it follows

$$
\frac{H_{n}}{m c^{2}}=\frac{1}{\cos \theta_{n}}-\alpha^{2}\left(\frac{a_{\infty}}{n a_{n}}\right)^{2}=\sqrt{1+\alpha^{2}\left(\frac{a_{\infty}}{n a_{n}}\right)^{2}}-\alpha^{2}\left(\frac{a_{\infty}}{n a_{n}}\right)^{2} .
$$

The expansion of this function in a series gives for circular orbits with $V_{n}=-e^{2} / r_{n}: \frac{H_{n}}{m c^{2}}=1-\frac{\alpha^{2}}{2}\left(\frac{a_{\infty}}{n a_{n}}\right)^{2}-\frac{\alpha^{4}}{8}\left(\frac{a_{\infty}}{n a_{n}}\right)^{4}+\cdots$. 
When $V_{n}=-e^{2} / r_{n}$ the Coulomb force does not have components in the additional subspace, and then everything $\frac{a_{n}}{a_{\infty}}=1$ and $\frac{H_{n}}{m c^{2}}=1-\frac{\alpha^{2}}{2 n^{2}}-\frac{\alpha^{4}}{8 n^{4}}+\cdots$ that agrees with the Bohr approximation, but not with the known fine structure formula [13].

However, in a multidimensional space the Coulomb potential must be a function of only the distance between the particles in this space. In addition, the electric field must be consistent with the Bio-Savart formula when applied to a full space. Therefore, the potential energy must have a form $V_{n}=-e^{2} / R_{n}$. Then for the same $r_{n}$ Formula (17) is specified: $e^{2} \frac{1}{R_{n}}=m c^{2}\left(\frac{n a_{n}}{r_{n}}\right)^{2}\left(1+\frac{a_{n}^{2}}{r_{n}^{2}}\right)^{-1 / 2}$, $\frac{n a_{n}}{r_{n}}=\frac{\alpha a_{\infty}}{n a_{n}}$,

$$
\frac{H_{n}}{m c^{2}}=\sqrt{1+\alpha^{2} \frac{a_{\infty}^{2}}{n^{2} a_{n}^{2}}}-\alpha^{2} \frac{a_{\infty}^{2}}{n^{2} a_{n}^{2}}\left(1+\alpha^{2} \frac{a_{\infty}^{2}}{n^{4} a_{n}^{2}}\right)^{-1 / 2} .
$$

Expanding this function in a series in powers, we find

$$
\begin{gathered}
\frac{H_{n}}{m c^{2}}=1-\frac{\alpha^{2}}{2} \frac{a_{\infty}^{2}}{n^{2} a_{n}^{2}}-\frac{\alpha^{4}}{2} \frac{a_{\infty}^{4}}{n^{4} a_{n}^{4}}\left(\frac{1}{4}-\frac{1}{n^{2}}\right)+\frac{\alpha^{6}}{8} \frac{a_{\infty}^{6}}{n^{6} a_{n}^{6}}\left(\frac{1}{2}-\frac{3}{n^{4}}\right)+\cdots \\
\frac{H_{n}}{m c^{2}} \approx 1-\frac{\alpha^{2}}{2 n^{2}}-\frac{\alpha^{4}}{2 n^{4}}\left(\frac{1}{4}-\frac{1}{n^{2}}\right)+\frac{\alpha^{6}}{8 n^{6}}\left(\frac{1}{2}-\frac{3}{n^{4}}\right)+\cdots .
\end{gathered}
$$

The Formula (19) agrees with the standard fine structure formula [13] and is some refinement of it. The transition of an electron from an orbit of the number $k$ to a lower orbit of the number $n$ leads to the emission of a photon of light energy $h v_{k n}=H_{k}-H_{n}$ at a frequency $v_{k n}$.

The Coulomb force of attraction between fixed in $X$ an electron and the proton, in the total space is equal to $e^{2} / R^{2}$. Projections of this force on subspaces $X$ and $Y$ are respectively equal $F_{\|}=\left(e^{2} / R^{2}\right) \sin \chi$ and $F_{\perp}=\left(e^{2} / R^{2}\right) \cos \chi$, where $\sin \chi=r / R$ and $\cos \chi=a / R$, so that $F_{\|}=e^{2} r / R^{3}=m c^{2} \alpha \frac{a_{\infty} r}{R^{3}}$ and $F_{\perp}=e^{2} a / R^{3}=m c^{2} \alpha \frac{a_{\infty} a}{R^{3}}$. When $r=0, \quad F_{\|}=0, F_{\perp}=e^{2} / a^{2}$. The corresponding dependences on $r / a_{\infty}$ are shown in Figure 3.

The Coulomb force in the six-dimensional space in the form $e^{2} / R^{2}$ is a consequence of applying the Bio-Savart formula to this space. According to this formula, the magnetic field of a charge $e$, stationary in the subspace $X$, at a distance $R$ from it in the total space is defined as $\boldsymbol{H}_{\mathrm{tot}}=\frac{e}{c R^{2}}\left[\boldsymbol{c} \boldsymbol{R}_{0}\right]$. Here $\boldsymbol{R}_{0}$ is the unit vector along the radius vector $\boldsymbol{R}$, $\boldsymbol{R}_{0}=\boldsymbol{r}_{0} \sin \chi+\boldsymbol{a}_{0} \cos \chi=\boldsymbol{r}_{0}(r / R)+\boldsymbol{a}_{0}(a / R), \quad \boldsymbol{r}_{0}$ is the unit vector along the radius vector $\boldsymbol{r}$ in $X, \boldsymbol{a}_{0}$ is the unit vector along the radius vector $\boldsymbol{a}$ in the plane of rotation in $Y$, and $c$ the velocity vector of the charge in $Y$, so that

$$
\boldsymbol{H}_{\mathrm{tot}}=\frac{e}{R^{2}}\left[\boldsymbol{c}_{0} \boldsymbol{R}_{0}\right]=\frac{e}{R^{2}}\left\{\left[\boldsymbol{c}_{0} \boldsymbol{r}_{0}\right](r / R)+\left[\boldsymbol{c}_{0} \boldsymbol{a}_{0}\right](a / R)\right\},
$$




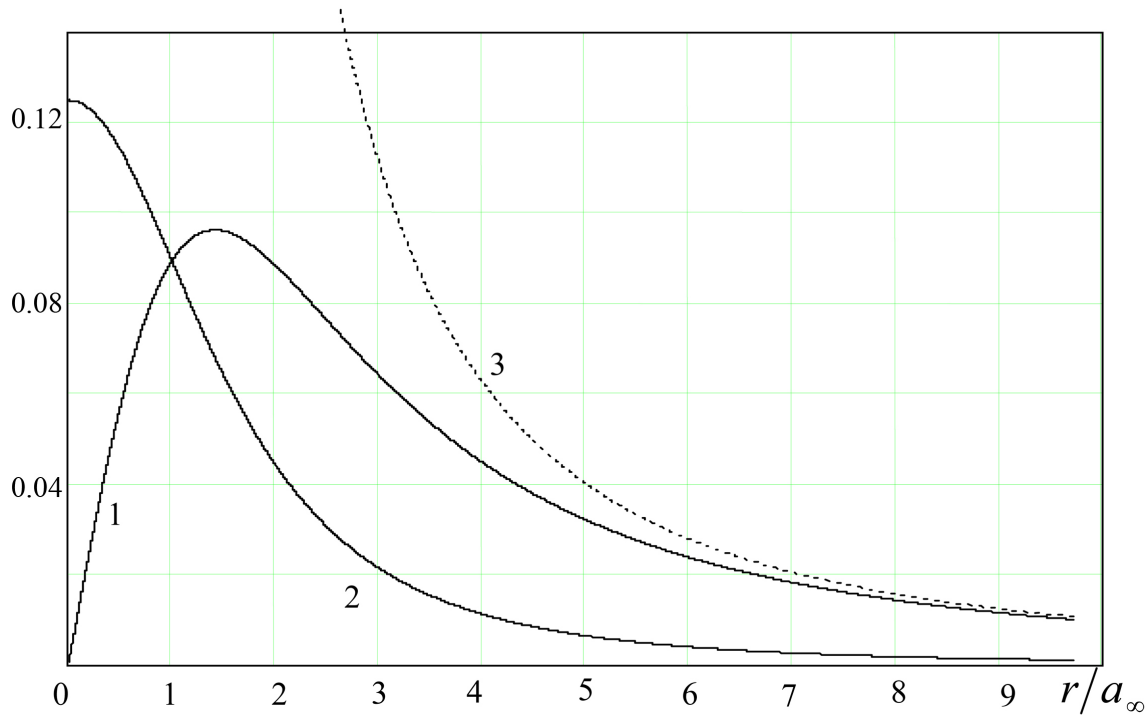

Figure 3. Projections of the Coulomb force on the observed and additional subspaces, depending on the normalized distance $r / a_{\infty}$ between the charges.

where $\boldsymbol{c}_{0}$ is a unit vector along the velocity $\boldsymbol{c}$.

Let us show that the Coulomb interaction force between two charges $e$ and $e^{\prime}$ is the Lorentz force $\boldsymbol{f}=\left(e^{\prime} / c\right)\left[\boldsymbol{c}^{\prime} \boldsymbol{H}_{\text {tot }}\right]$ acting on the charges moving in $Y$. According to (20), we have $\boldsymbol{f}=\left(e^{\prime} e / c R^{2}\right)\left\{\left[\boldsymbol{c}^{\prime}\left[\boldsymbol{c}_{0} \boldsymbol{r}_{0}\right]\right](r / R)+\left[\boldsymbol{c}^{\prime}\left[\boldsymbol{c}_{0} \boldsymbol{a}_{0}\right]\right](a / R)\right\}$ or

$\boldsymbol{f}=-\left(e^{\prime} e / R^{2}\right)\left\{\left[\boldsymbol{c}_{0}\left[\boldsymbol{c}_{0} \boldsymbol{r}_{0}\right]\right](r / R)+\left[\boldsymbol{c}_{0}\left[\boldsymbol{c}_{0} \boldsymbol{a}_{0}\right]\right](a / R)\right\}$, where $\boldsymbol{c}_{0}^{\prime}$ is the unit vector along the velocity $\boldsymbol{c}^{\prime}$. Expanding here the double vector products and taking into account the mutual perpendicularity of the vectors entering into them, we eventually get: $\left[\boldsymbol{c}_{0}\left[\boldsymbol{c}_{0} \boldsymbol{r}_{0}\right]\right]=-\boldsymbol{r}_{0},\left[\boldsymbol{c}_{0}\left[\boldsymbol{c}_{0} \boldsymbol{a}_{0}\right]\right]=-\boldsymbol{a}_{0}$,

$$
\boldsymbol{f}=\left(e^{\prime} e r / R^{3}\right) \boldsymbol{r}_{0}+\left(e^{\prime} e a / R^{3}\right) \boldsymbol{a}_{0}
$$

In the last formula, the first term represents the projection of the Coulomb force on $X$, the second term is its projection on $Y$. At $e^{\prime}=e$ their values, respectively, are equal to $F_{\|}$and. $F_{\perp}$. Hence it can be seen that the electric forces in $X$ are due to the motion of the charges in $Y$, in contrast to the magnetic forces in $X$, caused by the motion of charges in the same subspace $X$.

The electric force corresponding to the potential energy $V_{n}=-e^{2} / R_{n}$ is applicable in the balance of forces acting on an electron. In this case, the centrifugal force $p_{x} v / r$ and electric force $-e^{2} \frac{r}{R^{3}} \sqrt{1-\frac{v^{2}}{c^{2}}}=-e^{2} \frac{r}{R^{3}} \cos \theta$ act on the electron in the subspace $X$, where $R^{2}=r^{2}+a^{2},|e|$ is the charge of the nucleus of the atom, $p_{x}$ and $v$ accordingly the momentum and velocity of the electron, so that the balance of forces in $X$ is of the form

$$
p_{x} \frac{v}{r}=e^{2} \frac{r}{R^{3}} \cos \theta .
$$

Taking into account (1), (6), (12), $R_{n}=\sqrt{r_{n}^{2}+a_{n}^{2}}$ and (15), the Formula (21) is written in the form 


$$
\begin{gathered}
e^{2} \frac{r_{n}^{2}}{R_{n}^{3}}=m c^{2}\left(n y_{n}\right)^{2}, e^{2} \frac{1}{R_{n}}=m c^{2}\left(n y_{n}\right)^{2}\left(1+y_{n}^{2}\right), \\
e^{2} / r_{n}=m c^{2}\left(n y_{n}\right)^{2}\left(1+y_{n}^{2}\right)^{3 / 2}, y_{n}=a_{n} / r_{n} .
\end{gathered}
$$

The substitution $e^{2}=\alpha a_{\infty} m c^{2}$ in (23) gives

$$
\alpha \frac{a_{\infty}}{r_{n}}=\left(n y_{n}\right)^{2}\left(1+y_{n}^{2}\right)^{3 / 2},
$$

from which and from (12) it follows: $\frac{v_{n}^{2}}{c^{2}}=\sin ^{2} \theta_{n}=\alpha a_{\infty} \frac{r_{n}^{2}}{R_{n}^{3}} \cos ^{2} \theta_{n}$, $\tan ^{2} \theta_{n}=\alpha a_{\infty} \frac{r_{n}^{2}}{R_{n}^{3}}$.

Eliminating from this and from (12), we obtain

$$
n^{2} y_{n}^{2}=\alpha a_{\infty}\left(r_{n}^{2} / R_{n}^{3}\right) \text {. }
$$

With allowance for the motion of the electron in $X$ and equality $p \cos \theta=m c$, the component of the electric attraction force in $Y$ is equal to $F_{\perp} \cos \theta=\frac{e^{2} a}{R^{3}} \cos \theta=m c^{2} \alpha \frac{a_{\infty} a}{R^{3}} \cos \theta, \quad F_{\perp}=\frac{e^{2} a}{R^{3}}=m c^{2} \alpha \frac{a_{\infty} a}{R^{3}}$. It coincides in direction with the centripetal cosmological force $F=p c K_{0}$, where $p=\frac{m c}{\cos \theta}$, $K_{0}=\frac{\cos ^{2} \theta}{a_{\infty}}$ is the curvature of the helical trajectory of a free electron. By this $F=F_{0} \cos \theta$, where $F_{0}=\hbar c / a_{\infty}^{2}=m c^{2} / a_{\infty}$.

The balance of forces in the atom in $Y$ has the form $F_{0} \cos \theta+F_{\perp} \cos \theta=\frac{m c^{2}}{a} \cos \theta$, so $F_{0}+F_{\perp}=\frac{m c^{2}}{a}$, where $F_{\perp}=m c^{2} \alpha \frac{a}{R^{3}} a_{\infty}$. Hence we obtain $\frac{1}{a_{\infty}}+\alpha \frac{a_{n}}{R_{n}^{3}} a_{\infty}=\frac{1}{a_{n}}$,

$$
\alpha a_{\infty} \frac{a_{n}^{2}}{R_{n}^{3}}=1-\frac{a_{n}}{a_{\infty}}
$$

We divide (26) into (25). As a result, we find

$$
\frac{a_{n}}{a_{\infty}}=1-\frac{a_{n}^{4}}{r_{n}^{4}} n^{2} .
$$

Multiplying (25) by (27): we find the equation for $y_{n}=a_{n} / r_{n}$ :

$$
\begin{gathered}
n^{2} y_{n}\left(1-y_{n}^{4} n^{2}\right) R_{n}^{3} / r_{n}^{3}=\alpha, \\
\frac{\alpha}{n^{2}}=y_{n}\left(1+y_{n}^{2}\right)^{3 / 2}\left(1-y_{n}^{4} n^{2}\right), \\
\alpha^{2}=n^{4} y_{n}^{2}\left(1+y_{n}^{2}\right)^{3}\left(1-y_{n}^{4} n^{2}\right)^{2}, \frac{a_{n}}{a_{\infty}}=1-\frac{a_{n}^{4}}{r_{n}^{4}} n^{2} .
\end{gathered}
$$

Substitution $y_{n}^{2}=\frac{\alpha^{2}}{n^{4}} u_{n}$ gives $u_{n}=\left(1+\frac{\alpha^{2}}{n^{4}} u_{n}\right)^{-3}\left(1-\frac{\alpha^{4}}{n^{6}} u_{n}^{2}\right)^{-2}$. Up to terms 
of order $\alpha^{8}$ this is equal to $u_{n}=1-3 \frac{\alpha^{2}}{n^{4}} u_{n}+6 \frac{\alpha^{4}}{n^{8}} u_{n}^{2}-10 \frac{\alpha^{6}}{n^{12}}+2 \frac{\alpha^{4}}{n^{6}} u_{n}^{2}-6 \frac{\alpha^{6}}{n^{10}}$.

On the right-hand side of this equation we can put $u_{n}^{2}=1-6 \frac{\alpha^{2}}{n^{4}}$. Wherein

$$
\begin{gathered}
u_{n}=1-3 \frac{\alpha^{2}}{n^{4}}+2 \frac{\alpha^{4}}{n^{6}}+15 \frac{\alpha^{4}}{n^{8}}-24 \frac{\alpha^{6}}{n^{10}}-91 \frac{\alpha^{6}}{n^{12}}, \\
u_{1}=1-3 \alpha^{2}+17 \alpha^{4}-115 \alpha^{6}, u_{1}^{2}=1-6 \alpha^{2}+43 \alpha^{4}-332 \alpha^{6}+\cdots, \\
u_{n}^{2}=1-6 \frac{\alpha^{2}}{n^{4}}+4 \frac{\alpha^{4}}{n^{6}}+39 \frac{\alpha^{4}}{n^{8}}-60 \frac{\alpha^{6}}{n^{10}}-272 \frac{\alpha^{6}}{n^{12}}+\cdots, \\
\frac{a_{n}}{a_{\infty}}=1-\frac{a_{n}^{4}}{r_{n}^{4}} n^{2}=1-y_{n}^{4} n^{2}=1-\frac{\alpha^{4}}{n^{6}} u_{n}^{2} .
\end{gathered}
$$

According to (14) and $y_{n}^{2}=\frac{\alpha^{2}}{n^{4}} u_{n}$ we have $\frac{\sin ^{2} \theta_{n}}{\cos \theta_{n}}=\frac{\alpha^{2} u_{n}}{n \sqrt{n^{2}+\alpha^{2} u_{n}}}$, $H_{n}=m c^{2} \sqrt{1+\frac{\alpha^{2}}{n^{2}} u_{n}}+V_{n}$, where $V_{n}=-\frac{e^{2}}{R_{n}}$. Hence, from (22), (30) and $y_{n}^{2}=\frac{\alpha^{2}}{n^{4}} u_{n}$ we find: $V_{n}=-\frac{e^{2}}{R_{n}}=-m c^{2}\left(1+\frac{\alpha^{2}}{n^{4}} u_{n}\right) \frac{\alpha^{2}}{n^{2}} u_{n}$,

$$
\begin{aligned}
\frac{H_{n}}{m c^{2}} & =\sqrt{1+\frac{\alpha^{2}}{n^{2}} u_{n}}-\frac{\alpha^{2}}{n^{2}} u_{n}-\frac{\alpha^{4}}{n^{6}} u_{n}^{2} \\
& =1-\frac{\alpha^{2}}{2 n^{2}} u_{n}-\frac{\alpha^{4}}{8 n^{4}} u_{n}^{2}-\frac{\alpha^{4}}{n^{6}} u_{n}^{2}+\frac{\alpha^{6}}{16 n^{6}} u_{n}^{3}+\cdots \\
\frac{H_{n}}{m c^{2}} & =1-\frac{\alpha^{2}}{2 n^{2}}-\frac{\alpha^{4}}{2 n^{4}}\left(\frac{1}{4}-\frac{1}{n^{2}}\right)+\frac{\alpha^{6}}{2 n^{6}}\left(\frac{1}{8}-\frac{1}{2 n^{2}}-\frac{3}{n^{4}}\right) .
\end{aligned}
$$

According to (25), $\alpha$ is a function of the radii of the electron orbits in the subspaces $X$ and $Y: \alpha=\frac{n^{2} a_{n}}{r_{n}} \frac{a_{n}}{a_{\infty}}\left(1+\frac{a_{n}^{2}}{r_{n}^{2}}\right)^{3 / 2}$. Wherein $\alpha=\lim _{n \rightarrow \infty} \frac{a_{n}}{r_{n}} n^{2}$. Thus, the fine structure constant is determined by the ratio of the radii of the orbits in observed three-dimensional and additional subspaces.

It was noted in [13] that the virial theorem does not extend to quantum mechanics. However, it is not a matter of quantum mechanics in general, but in its relativistic case, when it is required to take into account the fundamental velocity $c$ in the formula for the kinetic energy of a particle. Indeed, the substitution $V_{n}$ in the proposed $-2 T_{n}$ for it in the Hamiltonian, where $T_{n}$ is the kinetic energy of the electron, $T_{n}=E_{n}-m c^{2}$, would give

$$
\begin{aligned}
H_{n} & =E_{n}-2 T_{n}=-E_{n}+2 m c^{2}=2 m c^{2}-m c^{2} \sqrt{1+\frac{\alpha^{2}}{n^{2}} u_{n}}, \\
\frac{H_{n}}{m c^{2}} & =1-\frac{\alpha^{2}}{2 n^{2}} u_{n}+\frac{\alpha^{4}}{8 n^{4}}\left(1-6 \frac{\alpha^{2}}{n^{4}}\right)-\frac{\alpha^{6}}{16 n^{6}}+\cdots \\
& =1-\frac{\alpha^{2}}{2 n^{2}}+\frac{\alpha^{4}}{8 n^{4}}+3 \frac{\alpha^{4}}{2 n^{6}}-15 \frac{\alpha^{6}}{2 n^{10}}-\frac{7 \alpha^{6}}{4 n^{8}}-\frac{\alpha^{6}}{16 n^{6}}+\cdots
\end{aligned}
$$


It can be seen from the Comparison (32) with (31) that in the Bohr approximation the virial theorem remains valid, but in the following order of expansion in powers of $\alpha^{2}$ the right-hand side of (32) deviates from (31) by an amount of order $\frac{\alpha^{4}}{4 n^{4}}+\frac{\alpha^{4}}{n^{6}}$.

The transition of an electron in the subspace $X$ of the $n$-th orbit into an orbit of a larger radius number $k$ is caused by the absorption of a photon of suitable energy.

The replacement in the Hamiltonian of the electron mass by its reduced mass [13] $m_{*}=m /\left(1+\frac{m}{m_{p}}\right)=\frac{m}{1.000544617031}$

takes into account the motion of the nucleus masses. In this case, the work function against the force of mutual attraction of the nucleus of the atom and the electron removed from the atom from the $\mathrm{n}$-th circular orbit is equal to

$$
A_{n}=m c^{2}-m_{*} c^{2}+m_{*} c^{2} \frac{\alpha^{2}}{2 n^{2}}+m_{*} c^{2} \frac{\alpha^{4}}{2 n^{4}}\left\{\frac{1}{4}-\frac{1}{n^{2}}-\frac{\alpha^{2}}{n^{2}}\left(\frac{1}{8}-\frac{1}{2 n^{2}}-\frac{3}{n^{4}}\right)\right\}+\cdots .
$$

Therefore,

$$
\begin{aligned}
A_{n}-A_{k}= & m_{*} c^{2} \frac{\alpha^{2}}{2}\left(\frac{1}{n^{2}}-\frac{1}{k^{2}}\right)+m_{*} c^{2} \frac{\alpha^{4}}{2}\left[\frac{1}{n^{4}}\left(\frac{1}{4}-\frac{1}{n^{2}}\right)-\frac{1}{k^{4}}\left(\frac{1}{4}-\frac{1}{k^{2}}\right)\right] \\
& -m_{*} c^{2} \frac{\alpha^{6}}{2}\left\{\frac{1}{n^{6}}\left(\frac{1}{8}-\frac{1}{2 n^{2}}-\frac{3}{n^{4}}\right)-\frac{1}{k^{6}}\left(\frac{1}{8}-\frac{1}{2 k^{2}}-\frac{3}{k^{4}}\right)\right\}+\cdots=h v_{k n}
\end{aligned}
$$

The transition of an electron from a higher orbit of the number $k$ to a lower orbit of the number $n$ leads to the emission of a photon with energy $h v_{k n}=H_{k}-H_{n}$ at a frequency $v_{k n}$.

The proton and neutron have the same sizes, and the nucleus is absent [14]. It's easy to explain. They consist of three quarks, although in different combinations. The proton consists of two $u$-quarks and one $d$-quark, the neutron consists of one $u$-quark and two $d$-quarks. Quarks move in the subspace $Y$ in their orbits of the Compton radius inversely proportional to the mass of the quark. The lightest quark is the $u$-quark. It determines the same size of the proton and neutron. Since the quarks move at a distance (Compton) from the center of rotation, in the center of the proton and neutron is a void. Since $u$-quark and $d$-quarks have opposite charges ( $2 e / 3$ and $-e / 3$ accordingly), the total charge of the neutron vanishes, but there is no reason for the zeroing of its resulting magnetic moment. The large magnetic moments of the proton and neutron are due to the relatively large magnetic moments of the constituent quarks [15].

In the six-dimensional treatment, the charges of particles and antiparticles are identical, but the sign of the magnetic field is determined by the direction of rotation of the particles in the additional space along the circumference of the Compton radius. The sign of the charge in this interpretation is just a mark corresponding to the direction of rotation. When time reversal (if reversal of time would possible), the particle moves in complete space reversely along the same 
trajectory as in the direct course of time. In this case, they automatically change to opposite signs of the fields, and the trajectory along the motion path turns out to be mirrored, and the particle acquires all the properties of the antiparticle.

\section{Conclusion}

It is shown that taking into account the motion of elementary particles in an additional subspace of a full six-dimensional space eliminates the need for the use of the wave function in quantum mechanics. In view of this motion, the fine structure formula for the circular orbits of an electron in a hydrogen atom is obtained and is refined. It is shown that the discreteness of the emission spectrum of an atom is due to the periodicity of the motion of its electron in the total space, when in the projection onto the observed and additional subspaces the electron commits an integer number of revolutions. A geometric interpretation of the fine structure constant in the form of a simple function of the ratio of the radii of the electron orbits in the observable and additional subspace is found.

\section{References}

[1] Weinberg, S. (2008) Cosmology. Oxford University Press, Oxford.

[2] Urusovskii, I.A. (2014) Physical Science International Journal, 4, 1110-1144. http://www.sciencedomain.org/issue.php?iid=564\&id=33

[3] Urusovskii I.A. (2015) Journal of Modern Physics, 6, 1220-1226.

[4] Urusovskii I.A. (2005) Physical Interpretations of Relativity Theory. Proceedings of International Meeting, 4-7 July 2005, BMSTU PH, Moscow, 318-326.

[5] Urusovskii I.A. (2012) Journal of Modern Physics, 3, 1749-1756. https://doi.org/10.4236/jmp.2012.311217

[6] Margolin, A.A. (1981) Khimiya i zhizn (Chemistry and Life), 9, 79. (In Russian)

[7] Klein, F. (1901) Uber neuere englische Arbeiten zur Gesammelte mathematische Aibhandlungen, B. 2. [On Recent English Works on the Collected Mathematical Acts, B. 2.] Springer, Berlin, 1922, 601 p.

[8] Klein, F. (1939) Higher Geometry. Gostekhizdat, Leningrad. 219 p. (In Russian)

[9] di Bartini, R.O. (1965) Reports of USSR Science Academy, 163, 861-865. (In Russian)

[10] Landau, L.D. and Lifshitz, E.M. (1976) Mechanics: Volume 1. 3rd Edition, Butterworth-Heinemann.

[11] Rumer, Y.B. (1956) Investigation in 5-Optics. Gostekhizdat, Moscow.

[12] Tamm, I.E. (1949) Fundamentals of the Theory of Electricity. Gostekhizdat, Moscow-Leningrad. (In Russian)

[13] Zommerfeld, A. (1951) Atombau und Spektrallinien. Band 2. Vieweg \& Sohn, Braunschweig, Friedr.

[14] Mostovoy, Y.A., Mouhin, K.N. and Tatarkin, O.O. (1996) Uspekhi Fizicheskikh Nauk, 166, 987-1022. (In Russian) https://doi.org/10.3367/UFNr.0166.199609f.0987

[15] Urusovskii I.A. (1999) Telecommunications and Radio Engineering, 6, 64-74. (In Russian) 